\title{
Propuesta de aculturación académica en la cátedra de Epistemología de la Licenciatura en Español y Literatura ${ }^{1}$
}

\author{
Rubén Darío Acero Vargas \\ Silvia Consuelo Morales Mariño**
}

* Magíster en Cooperación Internacional en Educación y Formación de la Universidad Paris V Descartes. Docente investigador de la Escuela de Idiomas de la Universidad Industrial de Santander, Bucaramanga, Colombia.

Correo electrónico:

rubendarioacerovargas1900@gmail.com.

** Magíster en Cooperación Internacional en Educación y Formación de la Universidad Paris V Descartes. Docente de la Escuela de Idiomas de la Universidad Industrial de Santander, Bucaramanga, Colombia. Correo electrónico:

silviaconsuelomorales@gmail.com.

Recibido: 06 de junio de 2017

Aprobado: 23 de octubre de 2017

Cómo citar este artículo: Acero Vargas, Rubén Darío, Morales Mariño, Silvia Consuelo. "Propuesta de aculturación académica en la cátedra de Epistemología de la Licenciatura en Español y Literatura". Rastros Rostros 19.35 (2017): 1-16. Impreso. doi: https://doi. org/10.16925/2382-4921.2017.35.03

\section{Resumen}

Introducción: este artículo tiene como objetivo describir el fenómeno de la aculturación académica en estudiantes de primer nivel de la Licenciatura en español y literatura de la Universidad Industrial de Santander (UIS). A su vez, propone una secuencia didáctica adaptada a las necesidades delimitadas en este proceso de aculturación desde la cátedra de Epistemología y el acompañamiento en el desarrollo de las competencias de lectura, escritura y oralidad. Esto con el fin de disminuir la deserción y la retención en la educación superior. Metodología: esta investigación es mixta con una metodología procesual participativa. Se emplea la investigación-acción y un enfoque socio-antropológico. Las teorías utilizadas se basan en los modelos epistemológicos de las ciencias sociales, las teorías de la argumentación, de la lectura, escritura y oralidad. Resultados: se constató que el acompañamiento del docente desde las herramientas empleadas y la interdisciplinariedad en la carrera ayudan a potenciar la adquisición de competencias de lectura, escritura y oralidad en estudiantes que, por lo general, llegan con vacíos conceptuales y procedimentales. Esto, en suma, contribuye a la aculturación académica. Conclusiones: en este proceso se identificó un choque inicial con la cultura a la que se llega, la puesta en marcha de estrategias individuales y la asimilación. Estas etapas varían de individuo a individuo y son el ritual de iniciación para comprender su paso por la universidad.

Palabras clave: aculturación académica, lectura, escritura, oralidad, universidad. 


\title{
Proposal for academic acculturation in the teaching of Epistemology for the Bachelor's degree of Spanish and Literature ${ }^{2}$
}

\begin{abstract}
Introduction: The objective of this article is to describe the phenomenon of academic acculturation with students of the first level of their Bachelor's Degree in Spanish and Literature of the "Universidad Industrial de Santander" (UIS). In turn, it proposes a didactic sequence adapted to the needs defined in this process of acculturation for the teaching of Epistemology and accompaniment in the development of reading, writing and oral skills; all of this with the aim of reducing desertion levels at university level education. Methodology: This research is mixed with a participatory process methodology. It uses investigative action with a socio-anthropological focus. The theories used are based on the epistemological models of the social sciences, the theories of argumentation, reading, writing $y$ oral skills. Results: It was found that the accompaniment of the teacher, with regards to the tools used and interdisciplinarity nature of the course, help to enhance the acquisition of reading, writing and oral skills in students that, in general, arrive with conceptual and procedural gaps. This process identified, an initial clash of cultures, the implementation of individual strategies and their assimilation. These stages vary from individual to individual and are the "right of passage" required to understand their path through university.

Key words: Academic Acculturation, Reading, Writing, Orality, university.

\section{Proposta de aculturação acadêmica na disciplina de Epistemologia da Licenciatura em Espanhol e Literatura ${ }^{3}$}

\section{Resumo}

Introdução: o artigo tem o objetivo de descrever o fenômeno da aculturação acadêmica em alunos do primer nível da Licenciatura em Espanhol e Literatura da Universidade Industrial de Santander (UIS). Também, propõe uma sequência didática adaptada às necessidades delimitadas ensse processe de aculturação a partir da disciplina de Epistemologia e o acompanhamento no desenvolvimento das competências de leitura, escrita e oralidade. Isso com o interesse de diminuir a deserção e a retenção na educação superior. Metodologia: a pesquisa é mista com uma metodologia processual participativa. Usa-se a pesquisa-ação e uma abordagem sócio-antropológica. As teorias utilizadas se baseiam nos modelos epistemológicos das ciências sociais, as teorias da argumentação, da leitura, da escrita e da oralidade. Resultados: constatou-se que o acompanhamento do professor a partir das ferramentas fornecidas e a interdisciplinaridade na graduação ajudam a potencializar a aquisição de competências de leitura, escrita e oralidade em alunos que, geralmente, chegam com vazios conceptuais e procedimentais. Isto, finalmente, contribui à aculturação acadêmica. Conclusões: no processo, identificou-se um choque inicial com a cultura à qual se chega, o início de estratégias individuais e a assimilação. Essas etapas variam de indivíduo a indivíduo e são o ritual de iniciação para compreender a passagem pela universidade.

Palavras-chave: aculturação acadêmica, leitura, escrita, oralidade, universidade.

\footnotetext{
2 This article is part of the research entitled "Diplomado en procesos didácticos de lectura, escritura y oralidad en educación superior" of the "Universidad Industrial de Santander", Colombia.

3 O artigo faz parte da pesquisa desenvolvida no "Diplomado em processos didáticos de leitura, escrita e oralidade em educação superior” da Universidade Industrial de Santander, Colômbia.
} 
La escritura alberga un potencial epistémico, es decir, no es sólo un medio de registro o comunicación, sino que puede ser un instrumento para revisar, transformar y acrecentar el propio saber. (Carlino 57).

\section{Introducción}

La integración a una nueva cultura siempre representa un reto para el "estudiante inmigrante". Aprender códigos explícitos e implícitos de las dinámicas humanas propias de una cultura determina el nivel de adaptación, apropiación y, por supuesto, de éxito en la misma. A su vez, el choque de la cultura de origen de los estudiantes y la cultura universitaria genera una multiplicidad de dinámicas que Arenas y Urzúa definen desde el enfoque sociológico del concepto de aculturación:

la aculturación tiende a inducir más cambios en el grupo no dominante (inmigrantes, minorías étnicas, religiosas, etc.) que en el grupo dominante (mayoría social), y puede ser reactiva (gatillar la resistencia al cambio en uno o ambos grupos), creativa (estimulando nuevas formas culturales que no se encuentran presentes en ninguna de las culturas en contacto), y retardada (iniciar cambios que se visualizan plenamente, años más tarde). (119)

Esto devela el proceso automático que se realiza semestre a semestre en la cultura universitaria en la que los estudiantes que buscan integrarse al grupo dominante académico utilizan estrategias de diferente tipo, algunas veces desde el rechazo a la nueva cultura, otras redefiniendo las interacciones de los miembros de cada cultura y, otras tantas, asimilando los nuevos valores culturales que se les presentan. Todo esto en procesos independientes que avanzan y retroceden a ritmos diferentes.

El presente artículo sistematiza y analiza el proceso de una secuencia didáctica desarrollada en la cátedra de Epistemología (3 horas semanales) con dos grupos de primer semestre (periodo 2016-II) de la Licenciatura en español y literatura (en adelante LEL) pertenecientes a la uis. Esta experiencia tiene como objetivo responder a la pregunta problema expuesta a continuación: ¿cómo potenciar la aculturación ${ }^{4}$

4 Este concepto es utilizado en varios paradigmas de las ciencias sociales para describir fenómenos con perspectivas diferentes. Sociológicamente el término es propuesto por Park y luego redefinido por Gordon. A su vez, en el Diccionario de la Real Academia universitaria desde los modelos epistemológicos de las ciencias sociales así como desde la lectura, la escritura y la oralidad en la academia?

Este proyecto se plantea ante la necesidad de evolución del carácter determinista de la universidad hacia una institución democratizada, en la que no solo se evalúe o juzgue, sino que también se enseñe a interpretar y a transformar el conocimiento, en que se valore la pluralidad de pensamientos. Tomando como punto de partida el perfil del egresado de LEL y los objetivos de aprendizaje del curso, el propósito de esta secuencia didáctica es transformar la enseñanza de la Epistemología, poniéndola al servicio de la investigación en educación. En ese sentido, se pretende que los estudiantes se inscriban en un modelo del conocimiento científico, comprendiendo los fundamentos y métodos de las ciencias humanas que facilitan su posicionamiento académico en las áreas específicas de su pregrado. Con esta secuencia didáctica se espera, entonces, sentar las bases de una conciencia investigativa en el estudiante y que este, a su vez, se convierta en un egresado que "comprende, critica e innova los procesos educativos de la lengua española, de literatura y de la comunicación humana" (UIs, perfil del egresado). Es decir, un profesional capaz de transformar la realidad educativa del país.

En particular, se busca que el conocimiento y análisis del discurso universitario y el acompañamiento proporcionado por el profesor en la comprensión y producción de textos académicos (orales o escritos), fundamentados en un modelo epistemológico, facilite su integración a la cultura académica. Por ello, los objetivos específicos que se proponen involucran la enseñanza de los modelos epistemológicos de las ciencias sociales y, a su vez, el desarrollo de un proceso de lectura, escritura y oralidad de textos académicos con el fin de que los estudiantes mejoren dichas competencias, para que comiencen su proceso de alfabetización académica ${ }^{5}$ (Carlino).

En lo que respecta a la población, es importante agregar que la uis, por ser una universidad de carácter público, recibe semestralmente una población estudiantil que proviene, en su mayoría, de una

de la Lengua Española (DRAE) conserva el sentido otorgado por los autores anteriores: aculturar se entiende como el proceso de "incorporar a un individuo o a un grupo humano elementos culturales de otro grupo".

5 "Señala el conjunto de nociones y estrategias necesarias para participar en la cultura discursiva de las disciplinas, así como en las actividades de producción y análisis de textos requeridas para aprender en la universidad" (Carlino 13). 
clase social media y baja. En relación con la muestra, los estudiantes de primer semestre de LEL son hijos de familias de clase obrera y campesinos, repartidos de manera equitativa entre los estratos 1,2 y 3 ; cuyo objetivo común es la ascensión social. Sus edades oscilan entre los 17 y los 27 años, siendo 19 el promedio del grupo. Son graduados, en la mayor parte de casos, de colegios públicos de la zona metropolitana, aunque también hay un gran porcentaje de estudiantes provenientes de otros municipios de Santander, e incluso de Colombia.

En términos de retención de los estudiantes en la universidad, en el Plan de Desarrollo Institucional UIS 2008-2018 se manifiesta lo siguiente: "se ha avanzado en la disminución de los niveles de retención de los estudiantes, experimentándose un aumento en el porcentaje de aquellos que cumplen exitosamente su ciclo de aprendizaje y se gradúan. Sin embargo, todavía se tiene un alto grado de retención entre los estudiantes" (UIS 37). En efecto, el porcentaje de estudiantes en retención por cohorte es del $23 \%$ según el mismo documento. Ese hecho justifica la necesidad de implementar más estrategias efectivas que ayuden a disminuir el porcentaje de retención en la universidad.

Ahora bien, la cultura universitaria semestre a semestre recibe nuevos estudiantes que requieren aculturarse y tener éxito en ella. El resultado de este intento deja una minoría victoriosa y un porcentaje importante fuera de la universidad. Así lo ratifica el postulado principal de Bourdieu y Passeron, en Los herederos (1964) y en la Reproducción (1970): las desigualdades reproducidas en la escuela se legitiman por medio de métodos y contenidos afines a la cultura de las clases dominantes. Por ende, en la universidad existen medios de clasificación vehiculados a través del lenguaje y, en suma, de una cultura académica. Sin embargo, como bien lo señala Carlino: "se trata de una cultura compleja, que suele permanecer implícita en las aulas universitarias" ("Escribir" 14). Lo que dificulta ampliamente la aculturación del estudiante.

Por otro lado, la escritura, la lectura y la oralidad, como medios emancipadores del hombre, constituyen hechos sociales (Durkheim) que hacen parte de la normalidad en la universidad, es decir, existen dos tipos de órdenes de hechos "los que son todo lo que deben ser y los que deberían ser diferentes de lo que son, los fenómenos normales y los patológicos" (Durkheim 91). En ese sentido, en el campo educativo, la escritura, la lectura y la oralidad son utilizadas continuamente como herramientas de segregación, lo que se alinea en el orden de hechos patológicos, alejándose del orden de hechos normales al que, en principio, debería pertenecer el ámbito universitario.

De igual manera, la disponibilidad de utilizar un código elaborado ${ }^{6}$ (Bernstein) por parte del "estudiante inmigrante", le da una gran ventaja frente al estudiante que domina un código restringido en la medida que el código elaborado, privilegiado en la universidad, requiere de una organización léxica y sintáctica mucho más compleja y variada, mientras que con un código restringido las estructuras utilizadas son mucho más rígidas y menos rigurosas. Lo que podría explicar por qué existe una brecha entre los individuos provenientes de medios sociales desfavorecidos con bajo capital cultural y económico (Bourdieu y Passeron) y los pertenecientes a medios sociales altos con uno mayor.

Para masificar el problema de la dificultad de adaptación a la vida académica universitaria se podría recurrir a otras teorías sociológicas como la socialización primaria o la socialización secundaria (Berger, Luckmann); sin embargo, es necesario aproximarse a los aportes de Carlino en Escribir leer y aprender en la Universidad para acercarse a una perspectiva pedagógica y lingüística de lo que implica enseñar a leer y escribir en una cátedra universitaria. Pues bien, si en primer lugar, el nuevo estudiante debe hacer un esfuerzo por adaptar las funciones que le otorga al lenguaje desde los ámbitos comunicativos, sociales y epistemológicos (Peña 1) a las exigencias de la universidad; en segunda medida, el profesor debe evitar reproducir los errores de la enseñanza habitual con el fin de disminuir la deserción en las aulas. Carlino plantea dos problemas que tiene dicha enseñanza tradicional. Por un lado, la falta de protagonismo del estudiante que asiste a una clase donde se le imparte una información sin permitirle intervenir activamente y, por otro, la necesidad de "enseñar los procesos y prácticas discursivas y de pensamiento que, como expertos desde un área, hemos aprendido en nuestros largos años de formación" (Carlino

\footnotetext{
6 "Se llamará "código elaborado" la forma del discurso por la cual es difícil de prever, considerando una serie de enunciados representativos, las elecciones sintácticas hechas por el locutor para estructurar su discurso, porque el sujeto ejecuta su selección a partir de una serie extendida de posibilidades que él puede organizar de una manera flexible" (Bernstein citado por Dantier 9).

7 "Es mucho más fácil prever, considerando una serie de enunciados representativos, las elecciones sintácticas que se realizan a partir de una serie limitada de opciones" (Bernstein citado por Dantier 9).
} 
"Escribir" 12). Es decir, es preponderante sentar las bases para que los estudiantes formulen sus propios modos de pensamiento a través de un metalenguaje requerido en la materia y de la construcción de un proyecto propio que los dirija hacia el aprendizaje significativo.

\section{Cuestiones Metodológicas}

Esta investigación es mixta con una metodología procesual participativa. El tipo es investigación acción con un enfoque socio-antropológico. Asimismo, se utiliza teoría fundamentada para la primera parte, a través de los cuatro modelos epistemológicos de las ciencias sociales primordiales para América Latina: la escuela crítica, la escuela hermenéutica, la escuela científica y las escuelas emergentes (teorías de la complejidad y las teorías constructivistas). Por el carácter procesual del proyecto se utilizan, para la fase de intervención, las teorías de la argumentación, de la lectura, escritura (transversalmente) y una metodología sucinta de investigación de las ciencias sociales.

La observación participante, desde el paradigma etnográfico, nutre y estructura la observación que se realiza en el aula de clase. La forma de observación etnográfica que se privilegió en este proyecto, fue la participación-observación, pues así el profesor en su función de miembro del grupo, adquiere cualidades de observador que interactúa con él obteniendo mayor información de la que podría obtener un observador externo; es decir, desde una observación etic, que deja de lado la construcción de símbolos que se encuentra en la observación emic que, por ende, se prioriza en este trabajo. "Emic y etic son, pues, 'dos puntos de vista' o perspectivas, que desde la óptica de Pike sólo significan dentro/fuera: la descripción émica o punto de vista del nativo, se sitúa dentro de la perspectiva del actor, mientras que la descripción ética o punto de vista del observador exterior, se sitúa fuera del agente" (Aguirre 85). Por esto, el proyecto usó las formas de observación emic y etic.

Uno de los riesgos en el análisis y recolección de información es el carácter participativo del maestro en el grupo, puesto que, si bien brinda una mayor comprensión de los símbolos internos de la muestra, puede propiciar una distorsión, debido a que su mirada unilateral impide una observación estructurada y adecuada. "Los inconvenientes o dificultades que se arguyen con más contundencia son: subjetividad del observador, canalizada en función de las características propias y las del (de los) sujetos(s) observado(s)" (Anguera 83). Es por esta razón que, en este trabajo, se propone la figura de un segundo investigador observador, quien desde su observación regula las posibles distorsiones de información de los fenómenos que se producen en el aula. A la vez que observa y evalúa el quehacer docente y su intervención en los procesos brindando un acercamiento de dos enfoques de observación, por un lado la autoevaluación del docente necesaria para asignar un valor determinado aciertos aspectos socio-pedagógicos relevantes e individuales del maestro, y la coevaluación por parte del segundo investigador observador quien regula y aporta una visión más homogénea y estructurada.

La forma que toma la descripción de los métodos, es decir, la observación que se realiza con la muestra de estudiantes, en esta investigación es la de secuencia didáctica puesto que, por su carácter secuencial, brinda en las diferentes etapas de observación la comprensión de momentos interrelacionados, que delimitan la temporalidad del corpus aquí analizado. “Asumir una 'unidad didáctica' como eje de observación se justifica en que cada uno de los momentos que la componen tienen gran variación en las características de la interacción y significación de cada proceso" (Rincón, Narváez y Roldán 17) Esto contribuye a realizar el desarrollo del método de forma organizada, a su vez, que esta organización estructura los momentos que componen la observación.

\section{Descripción de la secuencia didáctica.}

En este apartado se analiza el diagnóstico implementado y se describe el diseño general de actividades realizadas durante el semestre:

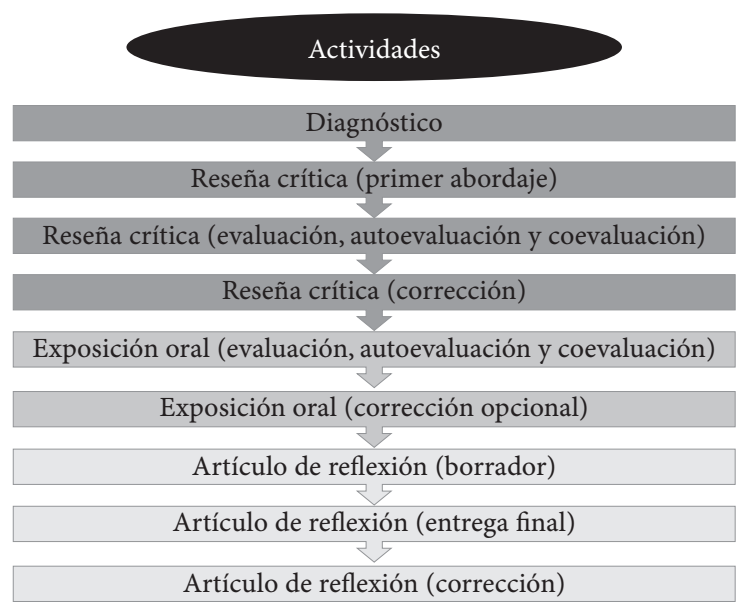

Figura 1. Diseño general de actividades. Fuente: elaboración propia. 
Nótese que la secuencia se divide en tres momentos clave: en verde, una fase exploratoria de la lectura y escritura donde prevalece la comprensión textual. En un segundo momento, un ejercicio oral de síntesis a partir de lecturas de fuentes secundarias y finalmente, una iniciación a la escritura académica a través de un artículo de reflexión, en el que se pone el relieve en el análisis crítico intertextual. Todas estas etapas tienen un espacio para la corrección del ejercicio.

\section{El diagnóstico.}

$\mathrm{Al}$ inicio del semestre, los estudiantes responden un diagnóstico con el que se busca determinar sus fortalezas y debilidades en cuanto a la escritura y lectura académica. Este taller es tomado del libro Didáctica de la comprensión y producción de textos académicos (Camargo , Uribe y Caro), pues el ejercicio se basa en los modelos de la comprensión lectora y se organiza en forma de secuencia didáctica con las siguientes fases: planificación de la lectura, focalización, supervisión de la eficacia del proceso y evaluación del proceso. Con este orden, se desea analizar la comprensión de la lectura y la producción escrita desde un antes, un durante y un después para así comprender el proceso individual de cada estudiante, y a su vez, el estado del grupo y la labor que se abordará con ellos.

Con este diagnóstico se encuentran problemas de diferente índole, que van desde la acentuación, puntuación y ortografía hasta dificultades de comprensión lectora mucho más profundos que se evidencian a través de las preguntas que demandan expresión escrita. También se aprecian falencias de estructuración en las estrategias que deben ser utilizadas en los momentos previos, durante y después de haber realizado la lectura.

A continuación, se presenta el análisis de la etapa final del diagnóstico realizado a los estudiantes en el cual se les solicita redactar un texto que exprese y justifique su opinión sobre la temática que se ha trabajado durante el diagnóstico, es decir, sobre la robótica, tópico predeterminado en el taller propuesto por Camargo, Uribe y Caro que corresponde a un artículo de opinión de corte expositivo e informativo, cuya temática es la evolución histórica de la robótica y algunas perspectivas basadas en postulados teóricos. El grado de complejidad es bajo, puesto que es un texto dirigido a un público general. Ahora bien, se escoge este punto ya que permite verificar, a partir de un ejercicio de redacción, las etapas previas a la lectura y durante la misma.
La pregunta tomada del diagnóstico para el análisis, es la siguiente:

en el artículo leído se esboza claramente la oposición entre los modelos de Kapek y Asimov. Escriba ahora un texto en el que exprese y justifique sus predicciones sobre el futuro de la robótica, en términos de su relación con el hombre. ¿Llegaremos a un día fatal en el que las máquinas dominen al hombre, o será posible el imperio de este amparado por las leyes de Asimov? (Camargo, Uribe y Caro 247)

A continuación, se transcriben las respuestas de cinco participantes, tomados al azar, codificados desde P1 hasta P5, con quienes se puede observar el estado de la comprensión y producción de lectura y escritura con el que inician los estudiantes de primer semestre que cursan la cátedra de Epistemología en el periodo académico 2016-II.

P1: Probablemente sí, más no habría o tendría certeza, es decir, quizás podrían estar las dos posiciones, puede que sí, como también puede que no, ya dependería de tan lejos quiera llegar la persona, como ser humano sin ayuda de una máquina "parlante".

P2: Sería algo destructivo para la sociedad porque acabarían con todos nosotros.

P3: Yo creo que es más acertada la idea de Asimov.

P4: La robótica seguirá avanzando y si no, se controla su difusión o dependencia, podríamos llegar a una catástrofe. No se (sic) si tan parecida a la que relata la ciencia-ficción; pero si se (sic) que esas tres leyes no son suficientes. (Actualmente y desde los inicios de estos hemos venido siendo reemplazados laboralmente por los mismos).

P5: La robótica en años muy próximos y a la implacable velocidad con que los avances en el conocimiento tecnológico avanza, terminará esclavo de su propios avances, hipnotizada por tanta facilidad que sus aparatos le brindan ahogandolo en una inestancable (sic) cantidad de posivilidad (sic), pues ellos se apoderan de cada acción humana referente al movimiento, haciendo reinar la madre parece por todo el mundo. Sí podría que las tecnologías en muy poco tiempo ya sean totales dueñas de nuestras vida e información, establecimiento un imperio con regimenes (sic) invisibles al ojo y sentido del ser humano. 
A partir de estos fragmentos se evidencian diferentes elementos que fueron relevantes en el desarrollo de la propuesta, puesto que, como se observa en las respuestas de los participantes, se obvian elementos clave en la comprensión de lectura, las estrategias previas, durante y posteriores a esta. Aquí, con el fin de expresar los elementos relevantes priorizados en el análisis de las respuestas anteriores se agregan los criterios establecidos por Garner y recopilados por Camargo, Uribe y Caro, que durante este proyecto fueron una fuente primordial para la implementación de estrategias de mejora de la acción pedagógica y, que a su vez, se utilizaron para evaluar la evolución de los estudiantes en el proceso de lectura. Dichos criterios son:

1. Establecimiento de un propósito de lectura.

2. Acceso al conocimiento previo para crear relaciones entre lo que ya se sabe y la nueva información que presenta el texto.

3. Generación y revisión de hipótesis y expectativas sobre los contenidos.

4. Anticipación de información.

5. Elaboración de interferencias necesarias para la comprensión del texto.

6. Focalización de la información más relevante del texto según sus características, al igual que del propósito y expectativas del lector.

7. Reconocimiento o establecimiento de las relaciones, explícitas o implícitas, entre los elementos formales de un texto (cohesión) y entre las ideas y conceptos (coherencia).

8. Organización de la información relevante en resúmenes, esquemas, cuadros sinópticos, mapas mentales o cualquier ordenador gráfico.

9. Integración de la información del texto con los conocimientos previos del lector. (Camargo, Uribe y Caro 230).

A continuación, se ven los criterios para enseñar y evaluar la expresión escrita propuestos por Camargo, Uribe y Caro (161), según el modelo constructivo-integrativo de Kintsch y Van Dijk:

A. Elaboración de una representación textual desde la microestructura o texto-base (macroproposiciones o ideas temáticas de cada párrafo) para establecer un orden o progresión temática entre las ideas, captando la relación que existe entre ellas.

B. Construcción de la macroestructura (macroproposición o idea general o global del texto), con el fin de reconocer el valor de cada una de ellas y determinar su nivel jerárquico.

C. Identificación de la superestructura (organización global del texto o estructura organizativa).

D. Aplicación de las macrorreglas o estrategias de omisión generalización, construcción.

E. Adecuación de los niveles de cohesión y coherencia en el texto-base.

F. Relación con los conocimientos previos, tanto en el primer momento de construcción de texto base, como en el segundo momento de integración textual.

G. Estructuración de la información: elaboración de esquemas.

H. Realización del resumen (atención a las ideas temáticas identificadas en el texto). (Camargo, Uribe y Caro 161).

Con base en los anteriores criterios se puede observar que los participantes P2 y P3 se limitaron a responder subjetivamente a la pregunta que contenía la consigna, lo que fue tendencia en gran parte de los diagnósticos a esta pregunta. Asimismo, se observa que P2: "Sería algo destructivo para la sociedad porque acabarían con todos nosotros" y P3: "Yo creo que es más acertada la idea de Asimov" son respuestas que no corresponden a los criterios que fueron determinados anteriormente: A, B, C, E, G y H. $\mathrm{La}$ intervención de $\mathrm{P} 1$ revela, de igual forma, una falta de estructuración $(G)$ y falta de estrategias de lectura como las relacionadas en los criterios 1, 2, 3, 5,8 y 9 , pues en la respuesta se observa una falta de análisis que son dados por la lectura previa, imprescindibles para responder a la cuestión de forma adecuada. Por otra parte, P4 y P5 son las respuestas más próximas a lo solicitado, aunque, la estructura no está muy definida, pues no es un texto en el que se pueda observar claramente una posición sobre el futuro de la robótica. Esto podría deberse a la vaguedad del término "texto" que no define el género textual que se solicita en el enunciado. A favor del planteamiento del diagnóstico se arguye que se explicita la orientación y los elementos que debe contener el "texto". Un aspecto común en los participantes, no sólo de P1 a P5, sino del grueso de la muestra, fue que, a partir de sus respuestas, se observa que no se comprendió adecuadamente la pregunta y lo que se les pedía elaborar en el punto del diagnóstico relacionado en este documento.

Dados los resultados anteriormente señalados, la propuesta que se formula busca integrar la lectura, escritura y oralidad para potenciar, especialmente, la 
producción escrita en el área de epistemología, a través de un artículo de reflexión en el cual los estudiantes expongan su posicionamiento epistemológico, construido por medio de lecturas, escritura de reseñas, exposiciones e investigaciones de fuentes primarias hechas a lo largo del semestre. Al respecto, el objetivo pedagógico principal es guiar al estudiante hacia una alfabetización académica desde la mirada de Carlino, es decir, brindarle las herramientas metodológicas y prácticas para el dominio del código discursivo en la cátedra de epistemología tanto desde la comprensión como desde la producción de textos orales y escritos, puesto que aprender a leer y escribir en un área del conocimiento es, por un lado, diferente a lo que aprendieron en bachillerato en la asignatura de Español y, por ende, requiere de una iniciación para la adquisición del nuevo discurso. Por otro lado, este es un proceso que debe entenderse como inacabado. La actualización permanente de las lecturas y la escritura son, entonces, requisito para seguir en el proceso de alfabetización académica y, de este modo, ingresar a la cultura de esta disciplina universitaria.

\section{La reseña crítica.}

Al inicio del semestre, se acuerda la lectura previa semanal de dos o tres capítulos de libros propiamente epistemológicos. Estos textos de profundización, además de aportar en términos de contenido a la cátedra, sirven para medir y mediar la comprensión y producción a través de un ejercicio de escritura: la reseña crítica. Con el fin de llevar a cabo el proceso, se les suministra unas pautas para escribir la reseña. De esta manera, se les indica cómo estructurar una reseña desde un antes, un durante y un después de la escritura. Además, se les entrega, al finalizar la clase, un formato de comprensión y producción escrita basado en las contribuciones de Camargo, Uribe y Caro. En la siguiente sesión, este taller se trae culminado con la intención de tener un insumo para participar en la socialización en clase y seguir nutriendo el texto. El diseño de esta guía comprende cuatro etapas: planificación de la lectura, focalización, supervisión de la eficacia del proceso y evaluación mismo (ver tabla 1).

Tabla 1. Reseña crítica

\section{Planificación de la lectura}

\begin{tabular}{ll}
\hline 1. Título, y autor & $\begin{array}{l}\text { - Epistemología de las ciencias sociales. Breve Manual. Capítulo III de Francisco Osorio } \\
\text { - Epistemología del Sur. Capítulo II de Boaventura de Sousa Santos }\end{array}$ \\
\hline 2. Motivación & Abordar conceptos propios de la epistemología en las ciencias sociales. \\
\hline 3. Objetivos & $\begin{array}{l}\text { - Comprender los modelos epistemológicos de las ciencias sociales. } \\
\text { los niveles inferencial y crítico-intertextual. }\end{array}$
\end{tabular}

4. Preguntas previas ¿Cómo se cumple con el objetivo pedagógico de transmitir al estudiante la construcción del objeto y que éste lo aprehenda a cabalidad sin que parta de ideas preconcebidas? Si somos ciegos ¿por qué es tan difícil aceptar la ceguera propia? ¿Qué es relevante?

\begin{tabular}{ll}
\hline 5. Conceptos clave & $\begin{array}{l}\text { Objeto de estudio, optimismo trágico, representación de los límites, representación distorsionada de } \\
\text { las consecuencias, conocimiento-emancipación conocimiento-regulación, perspectiva }\end{array}$ \\
\hline & \multicolumn{1}{c}{ Focalización } \\
\hline $\begin{array}{l}\text { 6. A qué tipología corres- } \\
\text { ponde cada texto }\end{array}$ & $\begin{array}{l}\text { a. Expositiva } \\
\text { b. argumentativa }\end{array}$ \\
& $\begin{array}{l}\text { c. narrativa } \\
\text { d. dialogal }\end{array}$ \\
\hline 7. Dentro de qué subgrupo se & a. Definición y descripción \\
ubicaría & b. Clasificación-tipología \\
& c. Comparación y contraste \\
& d. Problema-solución \\
e. Causa-consecuencia \\
f. Pregunta-respuesta
\end{tabular}

8. Cuál sería el tema central de cada libro. 
(viene)

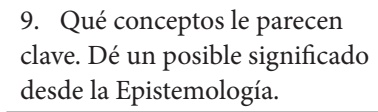

10. Escriba las ideas principales (de cada texto), no olvide subrayar en el texto.

\begin{tabular}{lc}
\hline \multicolumn{1}{c}{ Supervisión de la eficacia del proceso } \\
\hline 11. Escriba una reseña crítica & Espacio para escribir la reseña. Una página. \\
\hline & \multicolumn{1}{c}{ Evaluación del proceso } \\
\hline $\begin{array}{l}\text { 12. Qué puedes agregar luego } \\
\text { de la socialización del texto. } \\
\text { Qué relación encuentras entre } \\
\text { estos textos y los precedentes. }\end{array}$ \\
\hline
\end{tabular}

Fuente: adaptado de Camargo, Uribe y Caro (236).

Ahora bien, la planificación de la lectura aporta mayor claridad al estudiante que se enfrenta al texto y trata de darle un significado, siempre que se señale el propósito que cumple en la formación del estudiante. Carlino subraya que "uno de los procedimientos básicos para convertir en significado la información impresa es el muestreo o selección que realiza el lector, a distintos niveles textuales" ("Escribir" 70). En ese sentido, es clave que el profesor diseñe talleres en los que formule preguntas transversales y se brinde toda la información que considere oportuna para la correcta comprensión del texto. Por ello, en la primera parte de la guía que se les propone a los estudiantes se menciona información relevante sobre la obra: el título, el autor, la motivación para hacer la lectura, los objetivos y algunas preguntas previas para situarlos en la discusión epistemológica, así como los conceptos clave.

En el segundo segmento de la guía, es deber de los estudiantes analizar el texto en cuanto a su tipología textual (expositiva, argumentativa, narrativa, dialogal), el subgrupo al que pertenece (definición y descripción, clasificación-tipología, comparación y contraste, problema-solución, causa-consecuencia, pregunta-respuesta), el tema central de cada libro, las palabras que consideran clave, así como las ideas principales o que realicen un esquema. En la tercera fase, se supervisa la eficacia de las etapas previas planteadas en el formato, tomando como herramienta la reseña crítica. En esta deben, en un primer momento, condensar la información de los textos leídos en un resumen y en el último párrafo, exponer su opinión crítica. Es importante aclarar aquí que, de acuerdo a los criterios de evaluación, se precisa y explica al estudiante que este resumen se rige por las macrorreglas de Van Dijk: supresión, selección, generalización y construcción.

Durante la socialización en clase se discuten las lecturas, se releen algunos fragmentos y el docente expone los mecanismos para un mejor entendimiento del texto. Posteriormente, se abre el espacio a preguntas o reflexiones y se incita a los estudiantes a formular oralmente sus propias conclusiones. En este momento, se hace también una relación del texto actual con los precedentes y se añade una última reflexión. Esta fase, la evaluación del proceso, es una de las más importantes porque envía un mensaje intrínseco al estudiante. Todo lo que se lee, se habla y se escribe en clase es susceptible de ser asociarse con lecturas previas. La finalidad no es solo nutrir la comprensión, sino también facilitar la construcción de una postura epistemológica personal.

Luego de haber realizado un primer ejercicio de reseña con el que el estudiante comienza a comprender la lógica de las respectivas etapas de lectura y se enfrenta, por primera vez, a la producción escrita, se procede a elaborar una segunda reseña guiada que corresponde al primer ejercicio evaluativo. Los criterios, expuestos a continuación, son los que rigen la evaluación tanto de los estudiantes como del docente, y corresponden, también a una síntesis de los parámetros estudiados en clase para la elaboración de la reseña. 
Tabla 2. Criterios de evaluación de la reseña crítica

\begin{tabular}{|c|c|c|c|c|c|c|}
\hline & Criterios & 1 & 2 & 3 & 4 & 5 \\
\hline 1 & $\begin{array}{l}\text { Realiza las etapas previas a la escritura } \\
\text { de la reseña. }\end{array}$ & & & & & \\
\hline 2 & $\begin{array}{l}\text { La estructura del texto es clara: título, } \\
\text { presentación de la obra y de su autor, } \\
\text { resumen expositivo, comentario } \\
\text { crítico y conclusiones. }\end{array}$ & & & & & \\
\hline 3 & $\begin{array}{l}\text { Suprime la información irrelevante, } \\
\text { selecciona, generaliza la información } \\
\text { restante y construye un resumen } \\
\text { eficazmente. }\end{array}$ & & & & & \\
\hline 4 & $\begin{array}{l}\text { Desarrolla de manera coherente el } \\
\text { tema y el propósito del autor. }\end{array}$ & & & & & \\
\hline 5 & $\begin{array}{l}\text { No hace uso de la primera persona en } \\
\text { la reseña. }\end{array}$ & & & & & \\
\hline 6 & $\begin{array}{l}\text { Escribe un comentario crítico obje- } \\
\text { tivamente con argumentos y sin usar } \\
\text { juicios de valor de tipo: bueno, malo, } \\
\text { no me gustó. }\end{array}$ & & & & & \\
\hline 7 & $\begin{array}{l}\text { Relaciona el texto con lecturas prece- } \\
\text { dentes para comprender la epistemo- } \\
\text { logía de forma sistémica. }\end{array}$ & & & & & \\
\hline 8 & Utiliza adecuadamente los conceptos. & & & & & \\
\hline 9 & $\begin{array}{l}\text { Emplea los signos de puntuación } \\
\text { y el texto está escrito con buena } \\
\text { ortografía. }\end{array}$ & & & & & \\
\hline \multirow[t]{2}{*}{10} & $\begin{array}{l}\text { Hace un uso adecuado de los conec- } \\
\text { tores. }\end{array}$ & & & & & \\
\hline & Nota & & & & & \\
\hline
\end{tabular}

Fuente: elaboración propia.

Estos criterios son propuestos por el profesor y adjuntados al trabajo de los estudiantes para proceder a la autoevaluación, coevaluación y heteroevaluación. Las tres notas resultantes se suman y posteriormente se dividen en tres para así sacar el promedio de la calificación. Con esta medida cada nota equivale a un $33,3 \%$.

\section{Intervención oral planificada.}

Se considera lícito formular un ejercicio de oralidad para ayudar a la comprensión de los textos escritos pues "más que formas discursivas opuestas, el texto escrito y el discurso hablado funcionan de manera complementaria, cada uno con sus propias especificidades y funciones diferentes" (Peña 7). Este ejercicio de exposición interviene luego de la elaboración de las reseñas críticas pues los conceptos abordados y el discurso epistemológico en este estado del proceso ha debido sistematizarse en gran medida por los estudiantes. Los criterios que se acordaron para la evaluación son los siguientes:

Tabla 3. Criterios de evaluación de la intervención oral planificada

\begin{tabular}{|c|c|c|c|c|c|c|}
\hline & Criterios & 1 & 2 & 3 & 4 & 5 \\
\hline 1 & $\begin{array}{l}\text { Demuestra conocimiento y una visión } \\
\text { propia del tema. Utiliza adecuada- } \\
\text { mente los conceptos. }\end{array}$ & & & & & \\
\hline 2 & $\begin{array}{l}\text { El discurso oral evidencia una estruc- } \\
\text { tura que guía al enunciatario en el } \\
\text { desarrollo de las ideas. }\end{array}$ & & & & & \\
\hline 3 & $\begin{array}{l}\text { Suprime la información irrelevante, } \\
\text { selecciona, generaliza la información } \\
\text { restante y construye un discurso } \\
\text { eficazmente. }\end{array}$ & & & & & \\
\hline 4 & $\begin{array}{l}\text { Relaciona y ejemplifica el texto con } \\
\text { otros textos leídos en el curso o con } \\
\text { su realidad. }\end{array}$ & & & & & \\
\hline 5 & Respeta los tiempos establecidos. & & & & & \\
\hline 6 & $\begin{array}{l}\text { Interactúa adecuadamente con el } \\
\text { público. }\end{array}$ & & & & & \\
\hline 7 & $\begin{array}{l}\text { Utiliza un lenguaje adecuado a la } \\
\text { situación comunicativa (formal, téc- } \\
\text { nico, gramatical, sintáctico, semántico } \\
\text { y enunciatario). }\end{array}$ & & & & & \\
\hline 8 & $\begin{array}{l}\text { Presenta una dicción clara, sin } \\
\text { muletillas o barbarismos. Maneja una } \\
\text { velocidad y tono adecuado. }\end{array}$ & & & & & \\
\hline 9 & Mantiene una postura física adecuada. & & & & & \\
\hline \multirow[t]{2}{*}{10} & $\begin{array}{l}\text { Usa gestos que demuestran seguridad } \\
\text { en el tema. }\end{array}$ & & & & & \\
\hline & Nota & & & & & \\
\hline
\end{tabular}

Fuente: elaboración propia.

Por otro lado, la producción oral de las teorías y paradigmas epistemológicos se orientan a través de lecturas de fuentes primarias escogidas por ellos. Con esto identifican la bibliografía de los autores de su elección y, a su vez, ubican las obras en bibliotecas o medios virtuales para retomarlas en futuras lecturas.

\section{El artículo de reflexión}

La secuencia didáctica culmina con la redacción de un artículo de reflexión. Las lecturas, las reseñas críticas y el ejercicio de producción oral, realizados con antelación, sirven de insumo para la elaboración de este artículo que tiene como objetivo abordar los 
postulados epistemológicos del autor de su preferencia a la luz de una temática, problemática o ejemplo relacionado con la educación y, de este modo, reflexionar sobre los aportes conceptuales del escritor en el sistema educativo actual. A continuación, se presentan los criterios de evaluación:

Tabla 4. Criterios para el artículo de reflexión

\begin{tabular}{|c|c|c|c|c|c|c|c|c|c|}
\hline \multicolumn{10}{|c|}{ Citerios para el artículo de reflexión } \\
\hline 1 & Estructuración del artículo de reflexión. & 0.1 & 0.2 & 0.3 & 0.4 & 0.5 & 0.6 & & \\
\hline 2 & Emplear un lenguaje académico. & 0.1 & 0.2 & 0.3 & 0.4 & 0.5 & 0.6 & & \\
\hline 3 & Ortografía y puntuación. & 0.1 & 0.2 & 0.3 & 0.4 & 0.5 & 0.6 & & \\
\hline 4 & Dominio del tema. & 0.1 & 0.2 & 0.3 & 0.4 & 0.5 & 0.6 & 0.7 & 0.8 \\
\hline 5 & Nivel óptimo de contextualización. & 0.1 & 0.2 & 0.3 & 0.4 & 0.5 & 0.6 & 0.7 & 0.8 \\
\hline 6 & Coherencia para realizar relaciones intertextuales (otros autores). & 0.1 & 0.2 & 0.3 & 0.4 & 0.5 & 0.6 & 0.7 & 0.8 \\
\hline 7 & Desarrollo adecuado del método epistemológico. & 0.1 & 0.2 & 0.3 & 0.4 & 0.5 & 0.6 & 0.7 & 0.8 \\
\hline
\end{tabular}

Fuente: elaboración propia.

Estos parámetros se pactan en clase siguiendo un documento sobre los lineamientos para la redacción de un artículo de reflexión. De este modo, profesor y estudiantes deciden cuáles son los aspectos más relevantes y les otorgan un valor.

En el seguimiento del proceso, se acuerdan fechas y horas para las tutorías. En estas, los estudiantes formulan preguntas relativas al tema y a la redacción, se revisan los criterios para la entrega y dan cuenta de sus percepciones en esta etapa de construcción del borrador. Lo más importante aquí es que "sea el estudiante quien logre sus propias comprensiones, a partir de su experiencia y de su participación en los ámbitos provistos por el contexto académico, con una mínima intervención del tutor" (Escallón y Ferrero 116). En otras palabras, tanto el docente titular como el acompañante de la secuencia didáctica se enfocan exclusivamente en lo que el estudiante tiene para decir y, si es necesario, se realizan preguntas que le permitan reflexionar sobre la propuesta de escritura.

\section{Resultados}

Para describir los resultados específicos, se organizan en tres ejes: en lectura, oralidad, escritura y aculturación. Con esto se señalan los aspectos lingüísticos, por un lado, y el proceso de adaptación, por el otro.

\section{La lectura.}

Los jóvenes revelan, en espacios de diálogo como las tutorías con el profesor, la necesidad de recurrir a fuentes secundarias para llegar a la comprensión del texto demandado. Lo que no se descarta como inicio del proceso que se desarrolla en la materia y que los acerca a la complejidad de la lectura. Sin embargo, se les aconseja alejarse paulatinamente de segundas fuentes para que, al final del semestre, sean ellos quienes realicen sus propias interpretaciones y análisis acercándose de manera autónoma a los conceptos del autor. Pues como afirma Peña "el ejercicio de la lectura autónoma le permite al estudiante encontrarse directamente con los autores, sin la necesaria mediación del profesor; le enseña estrategias para autorregular su proceso de aprendizaje y lo prepara para continuar aprendiendo a lo largo de la vida" (3). A pesar de las indicaciones de no recurrir en exceso a estas fuentes, esta estrategia la utilizan reiteradamente tanto en la producción de las reseñas críticas como en la redacción del artículo de reflexión.

La búsqueda imperiosa de un autor que aborde el tema de un pensador reconocido a través de un lenguaje más sencillo demuestra la necesidad de la apropiación del metalenguaje empleado en el área y ese es uno de los grandes obstáculos que el estudiante debe franquear en el proceso de adaptación universitaria. "En Sousa la lectura era muy pesada, había mucho metalenguaje que no entendía muy bien y a la hora de avanzar se dificultaba y me tomaba mucho tiempo". Estas son las palabras de un estudiante en la encuesta final. Otro joven señala lo siguiente: "al principio me era muy difícil la comprensión de los textos. No conocía ningún concepto y me demoraba mucho". Aquí, el participante hace referencia a otro factor que 
les preocupa: el tiempo. En efecto, la disciplina de la lectura no es un hábito que se adquiere necesariamente en el colegio, lo que desemboca en la falta de tiempo para desarrollar las obligaciones de la carrera a cabalidad.

La falta de experiencia con géneros textuales similares en el colegio es señalada como otra causa de la dificultad inicial. "Al principio era un poco complejo comprender los textos, pero tras realizar las diferentes lecturas, uno se va acostumbrando. Comprender textos escritos fue algo complicado porque casi nunca, en el colegio, lo ponen a leer y escribir a uno artículos o reseñas". Esto revela la brecha entre los objetivos y competencias desarrolladas en las instituciones de educación media y las de educación superior. Con referencia a la educación en Colombia, Sanabria corrobora este planteamiento, pues asegura que "la lectura en el bachillerato está mal enfocada porque los estudiantes dedican todas sus materias a la lectura "obligatoria" de novelas, cuentos y poesía. En cambio la lectura de artículos y textos científicos es escasa" (15). En ese sentido, es necesario que los últimos grados colegiados preparen al estudiante para mitigar el efecto del acercamiento a la nueva cultura académica en la que entra.

Por otro lado, se presentan algunas imprecisiones en lo que los jóvenes consideran como un proceso de lectura. Por ejemplo, un estudiante relaciona la comprensión lectora solamente con la capacidad de imaginación. Respondiendo a qué estrategias ha usado para mejorar sus competencias en el curso de Epistemología, un estuante dice: "me ha ayudado usar la imaginación y así mejorar mi comprensión". En ese sentido, pareciera que él comprende la lectura como una comunión entre el texto y el lector, donde este último, gracias únicamente a su inventiva, genera comprensión. Se podría intuir, entonces, que él no tiene en cuenta los diferentes tipos y géneros textuales. Al respecto, Sanabria manifiesta que "el texto científico va dirigido al cerebro; el texto de fantasía va dirigido a despertar otras emociones, aunque a veces busca reacciones" (17). La lectura en la que el estudiante piensa es, tal vez, de carácter literario y no de textos académicos para los cuales es necesario, entre otras cosas, la capacidad de interrelacionar conocimientos previos más que la imaginación.

Del mismo modo, se comprende desde las diferentes intervenciones que el problema del acercamiento a los textos teóricos universitarios no es solo una cuestión de forma, sino de los estadios de comprensión que demandan los nuevos textos y la distancia de estos con el contexto de los estudiantes. Por esto, los procesos de lectura de cada materia deben ser guiados con objetivos específicos y claves que indiquen cómo se lee dicho texto desde cada asignatura. Claro está, sin restringir la interpretación individual de la obra. Esto es a lo que se refería Carlino con la labor del docente inclusivo, quien se debe preocupar por ofrecer categorías de análisis para la mejor comprensión de las lecturas, según su propia experiencia en la lectura de los textos de su disciplina.

En lo que se refiere al método evaluativo empleado (autoevaluación, coevaluación y heteroevaluación), se constata una inconsistencia logística que puede ser mejor pensada en próximas ocasiones. Dividir la nota en tres partes iguales supone que el juicio del profesor tiene solo 33,3\% de peso en la calificación final. Con ello, a pesar de las reflexiones acerca de la objetividad y la responsabilidad en las acciones, algunos estudiantes, motivados más por el deseo de aprobar que el de aprender, utilizaron la autoevaluación para otorgarse mucho más de lo que sus textos debían obtener.

\section{La oralidad.}

En la oralidad se hace evidente, por su parte, que el manejo del discurso, según la situación de comunicación de los estudiantes, es de carácter heterogéneo. Algunos presentan un mayor dominio del código académico mientras que otros manifiestan sendas dificultades en la capacidad de sintetizar un postulado y exponerlo con sus palabras. Esto puede deberse a que en la universidad, a diferencia del colegio, "la densidad y complejidad de los textos exigen del estudiante un mayor rigor y profundidad analítica" (Peña 3). Lo que, a su vez, implica que en el ámbito universitario se debe efectuar una serie de acciones autónomas, como la búsqueda de otras fuentes bibliográficas o bien el análisis concienzudo de un texto. La dificultad inicial para apropiarse del discurso epistemológico y hacer uso adecuado de la intertextualidad fue disminuyendo gradualmente. En el proceso, algunos grupos presentaron su exposición dos veces con el fin de corregir las falencias señaladas por sus compañeros y por el profesor.

La intervención oral planificada, aunque compleja, ayudó a los participantes de la muestra a exteriorizar y configurar con sus palabras el discurso epistemológico del autor que le correspondió, no sólo para la exposición oral, sino también para la escritura 
de su artículo de reflexión. Esto les permite un acercamiento preliminar a los planteamientos epistemológicos del autor y a la comprensión del discurso propio de la disciplina.

De este modo, cerca del final de la investigación, ellos aseguran, en la encuesta que se les suministró, haber obtenido una mejoría en la oralidad. "Al principio del curso fue muy complicada la comprensión de los textos, pero con las exposiciones no sólo aprendi a expresarme en clase, sino que también me aportó a la comprensión de lo que habíamos leído". En este momento, son capaces de reconocer los principales criterios para modular y enunciar un discurso con seguridad. Ahora le otorgan a la oralidad la capacidad para generar mayor grado de comprensión, a través de la organización y apropiación del discurso.

\section{La escritura.}

Para los participantes, la producción escrita representa un esfuerzo sustancial a lo largo del semestre. Desde el ejercicio de reseña crítica hasta el artículo de reflexión. En el primero, la dificultad para resumir lo esencial, construir un nuevo texto, exponer su opinión crítica y asociar a otros textos. En el segundo, el profundo análisis de una postura epistemológica, mediante la lectura de una fuente primaria y la relación de esta con una problemática de la educación para construir una reflexión académica.

En estos dos ejercicios en general, se han constatado problemas de redacción de todo tipo: abundantes faltas de acentuación (genesis, educacion, se hablara, elites, circulo, demostrare. Gomez, las criticas, se investigara, se analizara, demostrare, encerrara, conclusion, etcétera); algunos errores de puntuación como la posición incorrecta de la coma en los incisos explicativos; descuidos tipográficos como cabio en lugar de cambio; imprecisiones no solo en la conceptualización, sino también en los conectores utilizados, por ejemplo con base a en vez de con base en; falta de cohesión y coherencia entre las ideas "este libro se fundamenta en el obstáculo epistemológico y muestra sus múltiples clases, tanto cada una de las palabras como grupo nominal". Otro error común es el mal uso de los gerundios "sumando, también habla que debe haber una enseñanza socialmente activa”. Además, se encuentran oraciones sin verbo, redundancias, ejemplos fuera de contexto que no corroboran la afirmación inicial, etc.

Los estudiantes atribuyen estas falencias a la dificultad que presentan, en un primer momento, para comprender los textos científicos y luego para organizar ideas principales, secundarias y formular párrafos. A esta explicación se le agrega el desconocimiento del género académico; manifestado, por ejemplo, en la falta de reflexión y, en algunos casos, el tiempo transcurrido sin estudiar.

Este proceso de escritura es uno de los que se acompaña en mayor medida a lo largo del proceso, pero es en el que los resultados perceptibles no evidencian una evolución tan rápida. Se constata que aquellos estudiantes que realizaron de forma adecuada la reseña, de igual forma, desarrollan adecuadamente el artículo de reflexión, esto demuestra que a pesar de que estos dos géneros guardan diferencias sustanciales en forma y fondo, algunas habilidades de escritura son independientes del género textual.

Sin embargo, en lo referente a la escritura del artículo de reflexión hubo aspectos que fueron capitales en la correcta elaboración de la misma. En primer lugar, la participación en la tutoría fue determinante, pues la explicación personalizada de los objetivos del artículo de reflexión, el paso a paso para realizarla, así como la respuesta a inquietudes puntuales contribuyen al desarrollo adecuado del ejercicio. El objetivo era, como lo señala Carlino, "enseñar a estructurar las ideas por escrito según las particularidades de análisis y organización propias de un determinado dominio disciplinar" ("Escribir" 109), con el fin de que la construcción del borrador fuera el primer paso para la realización de un texto acorde con la asignatura, conceptualmente coherente y destinado a un lector crítico.

Por esto, no es sorprendente que quienes llevaban a la tutoría un borrador escrito y un plan de escritura, lograron concretar y mejorar sus artículos de reflexión. Por el contrario, aquellos que no participaron en la tutoría y no mostraron ningún tipo de borrador no evidencian en sus escritos la comprensión de la actividad que realizan. Ante la mejora gradual de este proceso, se les propone una nueva revisión del artículo de reflexión y posteriormente, la vinculación voluntaria a un semillero de investigación con propósitos de desarrollo del proceso de lectura y escritura, esto en aras de continuar y mejorar dichas competencias.

\section{La aculturación académica.}

La observación de la aculturación a través del seguimiento a los procesos de lectura, escritura, oralidad y de las dinámicas de aula arroja como resultado los planteamientos que se detallan a continuación, desde 
la aculturación como concepto de la antropología. En términos generales, se analiza el proceso del grupo, aunque se entiende que los procesos son individuales y cada estudiante requiere más, o menos, tiempo para lograr la adaptación académica.

El proceso de aculturación no es estático y atraviesa por diferentes fases, aunque no de forma secuencial. Si bien en un momento el estudiante puede abrazar la cultura meta, en otros puede rechazarla o generar resistencia a ella. (Prueba de inadaptación, es el caso de un estudiante que se limitó a copiar la reseña crítica de otro; creyendo, tal vez, que no sería descubierto ni penalizado). La conciencia de atravesar por ese proceso ayuda notoriamente a asumir las diferentes etapas evolutivas de la aculturación. De allí, la importancia del discurso que orienta el maestro en el que se hace consciente a los estudiantes sobre su proceso de integración y las aristas que este comprende.

El grupo se ha apropiado de algunas estructuras propias del pensamiento epistemológico, pues utiliza un metalenguaje próximo a la conceptualización que se realiza semana a semana, a través de las lecturas y su socialización. De igual forma, las relaciones intertextuales comienzan a ser de corte más académico, es decir, vinculan lecturas precedentes a la lectura de la semana, con lo que logran tener nuevos referentes distintos a su subjetividad para analizar y comprender los diferentes textos. El espíritu investigativo ha comenzado a forjarse en ellos. "Me parece interesante la idea de iniciar procesos de investigación para empezar a concebir textos y seguir haciéndolo luego cuando sea docente". Los motiva la posibilidad de mejorar el artículo para próximas publicaciones y comienzan a visionar la necesidad de ser investigadores.

La sensación de progreso en los participantes es generalizada. En la encuesta, varios participantes respondieron que el proceso les permitió mejorar sus competencias en lectura y escritura, así como comenzar a disfrutar este tipo de textos:

- "Considero que he mejorado mucho, ahora las lecturas las encuentro entretenidas y no son tan difíciles".

- "Al leer textos que antes no había leído, mi capacidad lectora de comprensión ha mejorado. Son textos complejos que ponen a la mente a trabajar más duro para lograr comprenderlos. Un ejemplo fue la reseña crítica, considero que mejoré bastante a la hora de la redacción".

- "Indudablemente esta clase me ha despertado un interés por los textos académicos, antes solo me gustaban las lecturas por placer, siento que este cambio de textos me ha ayudado mucho en mi vocabulario y mi manera de pensar".

- "Al leer este tipo de textos, me es necesario buscar conceptos y teorías, por lo tanto, he aumentado mi vocabulario y así mismo mi capacidad de análisis".

- "Con esta materia tengo una visión diferente, he mejorado mis capacidades intelectuales".

En consideración de estos comentarios y de lo que conversado en clase, es posible ver una mejora en la actitud de los estudiantes frente a la asignatura y, especialmente, frente a la comprensión y producción de textos académicos y científicos. Al comienzo del semestre, eran recurrentes las quejas por la extensión de las lecturas y el vocabulario lejano (dos estudiantes cancelaron el curso). Ahora, el grupo entiende con mayor claridad el objetivo de los textos en su formación y se comienza a empoderar del pensamiento epistemológico.

Un límite percibido en esta investigación, que juega un rol esencial, es el capital cultural de llegada de los estudiantes de primer semestre pues a partir de la observación parece determinante en el proceso de adaptación de los estudiantes. A menor capital cultural mayor dificultad para adaptarse a la cultura meta. Este tema es de gran interés y puede revelar un nuevo enfoque de análisis de estos procesos, sin embargo, acá no se desarrolla puesto que no responde al enfoque pedagógico de este proyecto sino a un enfoque más relativo a la sociología como tal. Para una próxima investigación es relevante estudiar el impacto de la cultura de origen sobre el proceso de adaptación e integración a la cultura meta.

\section{Conclusión}

Uno de los principales hallazgos, señalado por los mismos estudiantes, es la transversalidad en las cátedras del primer semestre de LEL. Ellos mencionan copiosamente que la relación de las temáticas y de los componentes de lectura, escritura y oralidad les ayuda, en gran medida, a la aceleración del proceso de adaptación. En efecto, las tutorías que se brindan en epistemología no son el único acompañamiento con el que cuentan los estudiantes, pues los docentes, el Programa de Inducción a la Vida Universitaria (PIVU) y el trabajo cooperativo entre ellos juegan a favor de la disminución del choque cultural y académico. 
Por otro lado, se evidencia que los estudiantes reconocen en este proceso una dinámica gradual que se nutre, esencialmente, de la dedicación y de la continuidad en el ejercicio lector y escritor. Esto representa un gran avance en la concepción de lo que implica ser estudiante universitario y futuro licenciado en español y literatura.

Ante la pregunta problema ¿cómo potenciar la aculturación universitaria desde los modelos epistemológicos de las ciencias sociales, así como desde la lectura, la escritura y la oralidad en la academia? Se plantea, al inicio de esta investigación, una metodología que conlleva desde el ejercicio pedagógico, no a una respuesta unilateral y positiva de cómo vincular a los estudiantes a la cultura académica universitaria, sino a una propuesta de acción pedagógica que se basa en planteamientos teóricos multi-paradigmáticos. Este proceso permite una reducción metódica y planificada de la gran brecha que se traza entre la cultura académica de la educación media y la de la educación superior. Desde la perspectiva antropológica de aculturación se erige el desarrollo de la investigación, así como desde tres competencias relativas a la aculturación académica universitaria: la lectura, la escritura y la oralidad.

En el proceso de lectura, se experimentó, en un primer momento, un choque provocado por el desconocimiento del género, del léxico y la falta de habituación a lecturas extensas. Este reto fue asimilado paulatinamente hasta ser aceptado, comprendido y, algunas veces, disfrutado. No obstante, en el proceso debieron recurrir a ciertas estrategias individuales que les sirvieron para afianzar su autonomía y responsabilidad, como los pasos propuestos en la guía del profesor, o bien para limitar su capacidad de análisis, como el uso de Wikipedia o resúmenes de las obras. Al final, de manera generalizada, reconocen la importancia de seguir el proceso sin tomar atajos y de continuar en el ejercicio activo de la lectura.

Por el contrario, en la escritura se observa un progreso menos marcado. La persistencia en faltas ortográficas o de coherencia y cohesión señalan la necesidad de seguir afianzando las técnicas de escritura, pues este es un ejercicio que requiere de más tiempo para ser pulido. Por esta razón, se invita a los estudiantes a participar en los semilleros existentes en la carrera y no desistir del proceso de producción escrita, que es por demás inacabado y requiere acompañamiento.

En cuanto a la oralidad, esta también representó un desafío, pero de igual manera, un camino al entendimiento de los temas epistemológicos y, especialmente, de lo que la cultura académica exige del orador. De este modo, tomaron conciencia acerca de lo que normalmente un profesor universitario espera de un estudiante cuando expone un planteamiento científico y pone en evidencia su opinión personal. De este modo, con estos conocimientos prácticos, se espera que su seguridad mejore en futuras intervenciones.

En general, el propósito de la aculturación a la academia fue asimilado por el grupo. Si algunos tenían dudas de por qué estaban en primer semestre de LEL, en este momento ven con más claridad los objetivos de su formación y conocen las competencias del perfil del egresado. La percepción del mundo universitario, desconocido por casi todos en un principio, fue agudizada y ampliada, lo que debería facilitarles ampliamente su paso por la universidad. Después del choque cultural del comienzo, la adaptación que experimentaron cargada de retrocesos y avances en su modo de comprender y producir, es el camino que les permitirá ser lectores y escritores autónomos y críticos. Es, en suma, un ritual de iniciación a la alfabetización académica.

\section{Referencias}

Anguera, María Teresa. “La observación participante”. Ángel Aguirre Baztán. Ed. Etnografía, metodología cualitativa en la investigación sociocultural. México: Marcombo, 1997. 73-84. Impreso.

Aguirre, Ángel. Etnografía, metodología cualitativa en la investigación sociocultural. México: Marcombo, 1997. Impreso.

Arenas, Pablo y Urzúa, Alfonso. "Estrategias de aculturación e identidad étnica: un estudio en migrantes sur-sur en el norte de Chile". Universitas Psychologica 15.1 (2016): 117-127. doi:10.11144/Javeriana.upsy15-1.eaie. En línea.

Bourdieu, Pierre y Passeron, Jean Claude. La reproducción. Elementos para una teoría del sistema de enseñanza. Buenos Aires: Siglo XXI Editores, 1996. En línea.

Los herederos. Los estudiantes y la cultura. Buenos Aires: Siglo XXI Editores, 2009. En línea.

Camargo, Zahyra; Uribe, Graciela y Caro, Miguel Ángel. Didáctica de la comprensión y producción de textos académicos. Quindío: Universidad del Quindío, 2009. Impreso. 
Carlino, Paula. "Enseñar a escribir en la universidad: cómo lo hacen en la universidad y por qué". Revista iberoamericana de educación 7.2 (2002): 43-61. En línea.

Escribir, leer y aprender en la universidad. Una introducción a la alfabetización académica. Buenos Aires: Fondo de Cultura Económica, 2009. Impreso.

Dantier, Bernard. Textes de méthodologie en sciences sociales choisis et présentés par Bernard Dantier, 2008. En línea.

Durkheim, Emilio. Las reglas del método sociológico. México: Fondo de Cultura Económica, 2001. En línea.

Peña, Luis Bernardo. "La competencia oral y escrita en la educación superior” Documento presentado al Comité Consultivo para la Definición de Estándares y Evaluación de Competencias Básicas en la Educación Superior, 2008. En línea.
Rincón, Gloria; Narváez, Elizabeth y Roldán Claudia. Interacción en el aula y lenguaje: ¿cómo enfrentar su investigación? Anagramas 4.7 (2005): 17-50. En línea.

Sanabria, Tyrone Emilio. La lectura del texto científico: un trabajo serio. Bogotá: Universidad Piloto de Colombia, 1997. Impreso.

Van Dijk, Teun. Texto y contexto. Semántica y pragmática del discurso. Madrid: Cátedra, 1980. En línea.

Universidad Industrial de Santander. Plan de desarrollo Institucional 2008-2018. Bucaramanga: UIS, 2007. En línea. 